\title{
Heulage und Co.: Was kann die Fütterung für die Lunge tun?
}

Ingrid Vervuert

\author{
Im Vordergrund der Prävention und Therapie chronischer Atemwegserkrankungen \\ stehen eine möglichst staubfreie Fütterung und Haltung. Als optimal gilt in vielen \\ Fällen die ganzjährige 24-stündige Weidehaltung, die jedoch nicht immer realisier- \\ bar ist. Doch welche positiven Effekte haben das Wässern oder Bedampfen von Heu \\ oder die Fütterung von Heulagen?
}

\section{Was ist eigentlich Stallstaub?}

Der Stallstaub stellt eine Mischung aus anorganischen Schmutzpartikeln, Mikroorganismen (Bakterien, Pilze, Hefen und mikrobielle Stoffwechselprodukte, wie z.B. Endotoxine oder Mykotoxine), Schadgasen (z. B. Ammoniak, Methan) und Pflanzenpartikeln dar, wobei als Eintragsquelle zu 80-90\% Futtermittel bzw. deren Umsetzungen verantwortlich sind. Hierbei darf auch die Einstreu nicht vergessen werden. Das Thema der Einstreuqualität ist ebenfalls Gegenstand der aktuellen Ausgabe.

Bei einem erhöhten Vorkommen von Stallstaub in der Umgebung von Pferden gelangen die Staubpartikel vermehrt in den oberen und unteren Atmungstrakt. Die sog. respiratorischen Partikel, die einen Durchmesser von $<5 \mu \mathrm{m}$ aufweisen, passieren dabei im Wesentlichen die terminalen Bronchiolen der Lunge mit Partikeldeposition, was als besonders gravierend bei der Entstehung des equinen Asthmas gilt. Als Abwehrreaktionen wird vermehrt Schleim produziert und verschiedene inflammatorische Reaktionskaskaden werden aktiviert.

In Anbetracht dessen, dass viele Pferde zumindest im Winter vielfach in Boxen gehalten werden, verdient die Stallluft deshalb besondere Aufmerksamkeit. Die Zusammensetzung des luftgetragenen Stallstaubs wird dabei maßgeblich durch die Auswahl und Hygiene der Futtermittel und Einstreumaterialien, den Ausscheidungsprodukten der Pferde über die Atmung, Haut, Kot und Harn sowie durch die Besatzdichte und das entsprechende Lüftungssystem des Stalls geprägt. Bei den täglichen Routinearbeiten, wie z.B. Füttern und Misten, kann ein erheblicher Anstieg der Staubkonzentration in der Stallluft in Abhängigkeit der Stallaktivität festgestellt werden. Daraus lässt sich u.a. die Forderung ableiten, dass Pferde möglichst zu solchen Zeiten nicht im Stall sind, wenn gemistet oder weitere Stallarbeiten, wie z.B. das Abladen von Heu oder Stroh, vorgenommen werden.
Zu beachten ist auch, dass die Staubkonzentrationen in der Umgebung des Pferdes, d. h. im Kopf- und Nüsternbereich durch die Heu- oder Strohaufnahme deutlich höher sind (> 7-fach) als die Staubgehalte in der Stallluft auf der Stallgasse außerhalb der Box. In Geflügelställen gibt es Bestrebungen, die Staubpartikel durch den Vorgang der Ionisation elektrostatisch zu verändern, um somit die Bildung größerer Partikelzusammenlagerungen zu erzielen, die gemäß der Gravitation schneller absinken, um somit das Vorkommen der respiratorischen Partikel zu mindern. Diese Maßnahme führt aber in Pferdeställen nicht zu dem gewünschten Erfolg, sodass der Fokus auf die Reduzierung des Staubeintrags durch Fütterung und Haltung gelegt werden sollte.

\section{Heu als Staubeintragsquelle?}

Eine nicht unerhebliche Staubbelastung stellt die Fütterung von Heu dar, da selbst bei hygienisch einwandfreien Qualitäten eine 4-6-mal höhere Staubentwicklung im

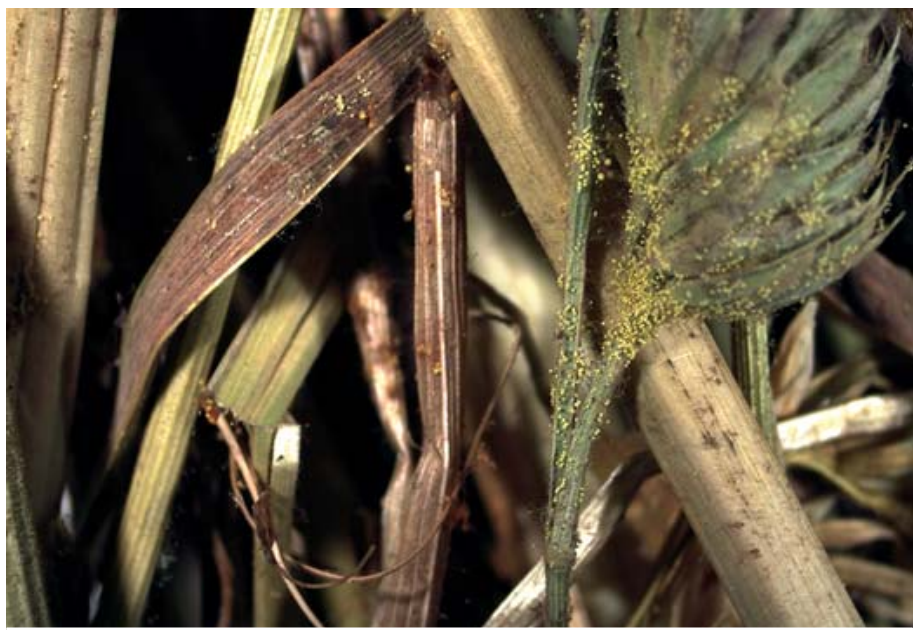

- Abb. 1 Mikrobiell kontaminiertes Heu (10-fache Lupenvergrößerung). (c) I. Vervuert 
- Tab. 1 Orientierungswerte für produkttypische und verderbanzeigende Mikroorganismen in Raufuttermitteln (Angaben in Kolonie bildenden Einheiten [KbE] pro g Futter) [1].

\begin{tabular}{|c|c|c|c|c|c|c|c|}
\hline \multirow[b]{2}{*}{ Keimgruppe } & \multicolumn{3}{|c|}{ Mesophile aerobe Bakterien $\times 10^{6} \mathrm{KbE} / \mathrm{g}$} & \multicolumn{3}{|c|}{ Schimmel- und Schwärzepilze $\times 10^{3} \mathrm{KbE} / \mathrm{g}$} & \multirow{2}{*}{$\begin{array}{l}\text { Hefen } \times 10^{5} \mathrm{KbE} / \mathrm{g} \\
7\end{array}$} \\
\hline & 1 & 2 & 3 & 4 & 5 & 6 & \\
\hline Heu & 30 & 2 & 0,15 & 200 & 100 & 5 & 1,5 \\
\hline Stroh & 100 & 2 & 0,15 & 200 & 100 & 5 & 4 \\
\hline
\end{tabular}

Keimgruppe 1: produkttypische Keime, z. B. Gelbkeime;

Keimgruppe 2: verderbanzeigende Keime Bacillus, Staphylococcus, Micrococcus; Keimgruppe 3: verderbanzeigende Keime Streptomyceten;

Keimgruppe 4: produkttypische Schimmel- und Schwärzepilze, z. B. Fusarium spp.;

Keimgruppe 5: verderbanzeigende Schimmelpilze, z. B. Aspergillus, Penicillium, Scopulariopsis, Wallemia;

Keimgruppe 6: verderbanzeigende Hefen

Vergleich zu staubarmen Konfektionierungen, wie z.B. Heupellets, beobachtet werden können.

Noch gravierender wirken sich solche Futterqualitäten aus, die Mängel im Hygienestatus aufweisen. Hier sind häufig Heu- und Strohqualitäten auffällig, die aufgrund von schlechten Ernte- oder Lagerungsbedingungen erhöhte Bakterien- und Schimmelpilzgehalte aufweisen $(\triangleright$ Abb. 1 und 2 ).

Generell muss unterstrichen werden, dass Futtermittel auch ohne nachteilige Abweichungen in der hygienischen Qualität ebenfalls nicht keimfrei sind [1] und dass eine produkttypische Keim- und Pilzflora eine Schutzbarriere gegenüber unerwünschten Keimen darstellen kann ( $\triangleright$ Tab. 1).

Das Spektrum der Abweichungen in den Futtermitteln, die maßgeblich die Staubbelastung in der Umgebung des Pferdes erhöhen, ist sehr vielfältig; zu den wichtigsten Veränderungen in den Futtermitteln zählen Milben, erhöhte Gehalte an Bakterien und -toxinen, Schimmelpilze und -toxinen sowie Hefen. Alarmierend ist das Ausmaß der beanstandeten Futtermittelproben bei den entsprechenden Untersuchungen. Zahlreiche Veröffentlichungen aus den letzten 10 Jahren belegen, dass bis zu $84 \%$ der untersuchten Strohproben massive Gehalte an Bakterien und Schimmelpilze aufweisen, beim Heu wurden rund $50-70 \%$ der überprüften Proben mit erhöhten Keim- und Pilzgehalten beanstandet.

\section{Woran erkennt man hygienisch bedenkliche Heu- und Strohqualitäten?}

Bei schlechten Heu- oder Strohqualitäten fällt beim Aufschütteln eine deutlich erkennbare Staubentwicklung auf, die als Indiz für einen erhöhten Besatz mit Schmutzpartikeln sowie Bakterien und Schimmelpilzen gewertet werden kann. Des Weiteren weisen solche Heu- und Strohqualitäten z. T. eine gräuliche Farbe auf und die Fut-

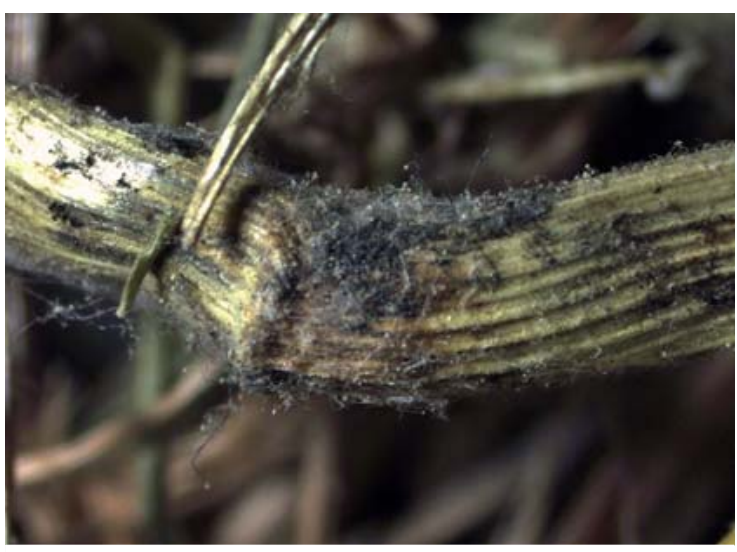

Abb. 2 Mikrobiell kontaminiertes Heu (10-fache Lupenvergrößerung). ㅇ I. Vervuert

termittel riechen muffig oder staubig. Anzumerken ist allerdings auch, dass selbst sensorisch einwandfreie Heuqualitäten eine hohe mikrobielle Kontamination aufweisen können.

Neben der regelmäßigen sensorischen Überprüfung der eingesetzten Futtermittel ist die mikrobiologische Untersuchung der Raufutter vielfach sinnvoll, um den Grad der Kontamination mit Bakterien und Schimmelpilzen besser einschätzen zu können [1] ( Tab. 1).

\section{Wässern von Heu?}

Eine Möglichkeit, den Staub im Heu zu binden bzw. teilweise auszuwaschen, ist das Wässern des Heus. Allerdings ist die einwandfreie hygienische Qualität des Heus eine wichtige Voraussetzung, da Bakterien, Schimmelpilze und Hefen in den Magen-Darm-Trakt gelangen, was u.U. zu gastrointestinalen Störungen führen kann.

Grundsätzlich gilt für das Wässern von Heu, dass ein 10minütiges vollständiges Eintauchen von Heu, d.h.z.B. $5 \mathrm{~kg}$ Heu in 30 I Wasser bei einer Wassertemperatur von 
- Tab. 2 Bakterien und Pilze in Abhängigkeit der Bedampfungstechnik (Mittelwerte für 5 verschiedene Heuqualitäten in KbE pro g Futtertrockenmasse) [2].

\begin{tabular}{|l|c|c|c|}
\hline Parameter & trockenes Heu & Bedampfen mit Eigenkonstruktion & Bedampfen mit kommerziellem Gerät \\
\hline Bakterien & 234422 & 549540 & 12 \\
\hline Pilze & 53703 & 5012 & 5 \\
\hline
\end{tabular}

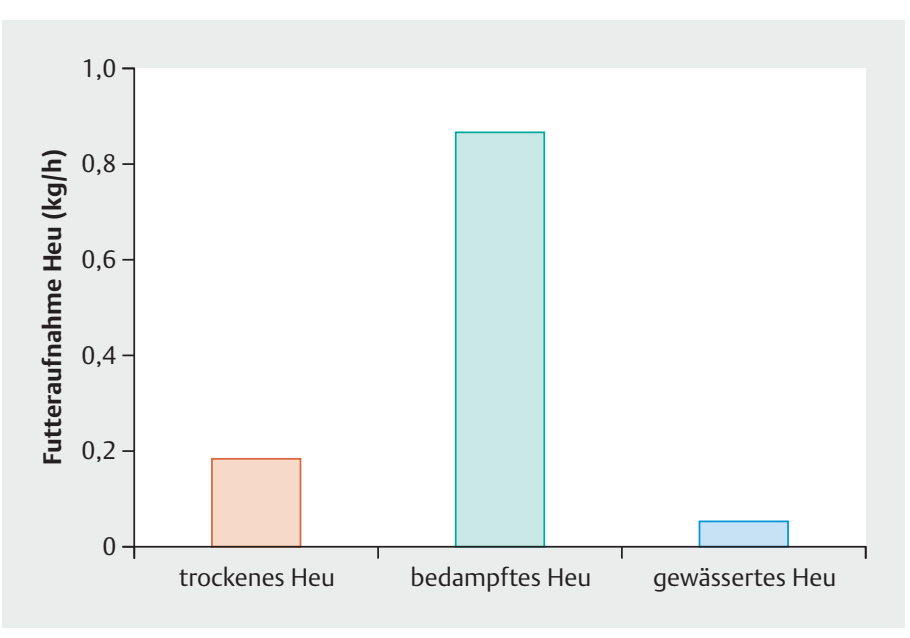

- Abb. 3 Futteraufnahme $[\mathrm{kg} / \mathrm{h}]$ in Abhängigkeit der Heubehandlung bei Pferden [3]. () I. Vervuert

\section{Akzeptanz?}

Neben dem erheblichen Arbeitsaufwand und dem vielfach nicht sachgerechten Wässern des Heus stellt aber die zum Teil schlechte Akzeptanz des gewaschenen Heus ein Problem in der Fütterung von Pferden mit chronischen Atemwegserkrankungen dar. In einem Wahlversuch konnte bei Polopferden gezeigt werden, dass gewässertes Heu fast vollständig von den Pferden gemieden wurde, wenn Alternativen wie bedampftes Heu oder trockenes Heu zur Verfügung standen [3] ( $\triangleright$ Abb. 3).

\section{Bedampfen von Heu?}

Seit einigen Jahren wird als Alternative zum Wässern das Bedampfen des Heus empfohlen. In verschiedenen Untersuchungen konnte dabei gezeigt werden, dass das Bedampfen von Heu mit dem Erreichen von Temperaturen im Heu bis zu $>99^{\circ} \mathrm{C}$ zu einer deutlichen Reduktion der mikrobiellen respiratorischen Partikel führte [2].

\section{Temperatur entscheidend}

$16^{\circ} \mathrm{C}$ ausreicht, um eine fast vollständige Reduktion der lungengängigen Partikel zu bewirken [2]. Das Waschwasser sollte nach jedem Heuwässern entfernt werden, um eine mikrobielle Anreicherung zu vermeiden. Ein längeres Eintauchen des Heus (>30 min) hat insbesondere bei hohen Außentemperaturen zur Folge, dass ein massives Vermehren von Mikroorganismen möglich ist. Umgekehrt wird auch häufig der Fehler gemacht, dass das Heu nur unzureichend mit Wasser benetzt wird (z. B. Anfeuchten mit Blumenspritze oder Gießkanne), sodass keine ausreichende Auswaschung der Mikroorganismen erfolgt.

\section{Nährstoffzusammensetzung?}

Neben dem Auswaschen der Mikroorganismen gehen auch Mineralstoffe und wasserlösliche Kohlenhydrate bis zu 50\% durch das Wässern des Heus verloren. Ausgewaschene Mineralstoffe können allerdings durch die Zulage eines Mineralfutters vollständig kompensiert werden und die Reduktion von wasserlöslichen Kohlenhydraten ist z. B. bei adipösen Pferden eher wünschenswert.

Merke

Für das Wässern von Heu ist ein 10-minütiges vollständiges Eintauchen von Heu, d.h.z.B. 5 kg Heu in 30 I Wasser bei einer Wassertemperatur von $16^{\circ} \mathrm{C}$, ausreichend.
Kommerzielle Heubedampfer sind dabei deutlich effektiver in der Reduktion des mikrobiellen Besatzes als selbstgebaute Konstruktionen ( $\bullet$ Tab.2), was vermutlich mit einer nicht-vollständigen Penetration des heißen Wasserdampfs im Heu bei selbstgebauten Bedampfern zusammenhängt [2]. Gerade thermophile Bakterien können sich bei Temperaturen zwischen $40-70^{\circ} \mathrm{C}$ im Heu optimal vermehren, sodass eine unzureichende Penetration des heißen Wasserdampfs mit mittleren Temperaturen im Heu von $58^{\circ} \mathrm{C}$ zu einer Vermehrung von Mikroorganismen im Heu führen kann [2].

Durch die Reduktion der Keimgehalte kommt es durch das Bedampfen von Heu zu einer nachweisbaren Reduktion der inhalierbaren Partikel, ein Effekt, der für die Vermeidung chronischer Atemwegserkrankungen von wesentlicher Bedeutung ist.

\section{Nährstoffzusammensetzung?}

Im Gegensatz zum Wässern des Heus kommt es durch das Bedampfen nicht zu einer Reduktion der Mineralstoffe, wohingegen ebenfalls Verluste bei den wasserlöslichen Kohlenhydraten um 18\% durch das Bedampfen des Heus auftreten können [2].

Aktuell ungeklärt bleibt die Frage, welche Bedeutung die hohen Wasserdampftemperaturen (bis zu $>99^{\circ} \mathrm{C}$ ) für die 
Proteinqualität im Heu besitzen. Bei diesen Temperaturen muss mit Denaturierungsprozessen des Proteins gerechnet werden. Inwieweit diese Veränderungen die Protein- bzw. Aminosäuren-Versorgung des Pferdes nachteilig beeinflussen können, kann zum aktuellen Zeitpunkt nicht eindeutig beantwortet werden.

\section{Haltbarkeit?}

Weitere Fragen beschäftigen sich mit der Haltbarkeit des Heus nach dem Bedampfen. Hierzu konnte prinzipiell gezeigt werden, dass bis zu $24 \mathrm{~h}$ nach dem Bedampfen keine erneute nennenswerte mikrobielle Kontamination des Heus auftrat, allerdings lassen die Untersuchungen offen, wie das bedampfte Heu gelagert wurde (Laborbedingungen vs. Stall?), sodass hieraus aktuell keine eindeutige Empfehlung zur Haltbarkeit von bedampften Heu abzuleiten ist.

\section{Besser als ihr Ruf: Heulagen!}

Eine weitere Möglichkeit zur Reduktion der inhalierbaren Staubpartikel ist die Fütterung von Heulagen (Trocknungsgrad zwischen 50-85\%) oder Grassilagen (Trocknungsgrad < 50\%). In zahlreichen Studien konnte der Fütterungswechsel von Heu auf Heulage zu einer signifikanten Verbesserung des klinischen Status sowie von Lungenfunktionsparametern bei chronischen Atemwegserkrankungen gemessen werden.

In den letzten Jahren ist allerdings die Fütterung von Heulagen durch die populärwissenschaftlichen Medien in Misskredit gekommen, was u.a. auf der pauschalen Annahme basiert, dass die Fütterung von Heulagen oder Grassilagen eine „Dickdarmübersäuerung“ beim Pferd induziert. Heulagen wie sie typischerweise beim Pferd gefüttert werden, besitzen ähnliche $\mathrm{pH}$-Werte wie Heu, nur Grassilagen, die kaum beim Pferd zum Einsatz kommen, besitzen einen $\mathrm{pH}$-Wert $<5$.

\section{Nährstoffzusammensetzung?}

Betrachtet man die Nährstoffzusammensetzungen von Grassilagen, Heulagen oder Heu, die von identischen Flächen gewonnen wurden ( $\bullet$ Tab. 3), so fallen im Wesentlichen geringere Gehalte an wasserlöslichen Kohlenhydraten in der Grassilage auf, da durch die Fermentationsprozesse während des Silieren die wasserlöslichen Kohlenhydrate abgebaut werden.

Weitere wesentliche Unterschiede im Nährstoffprofil können vielfach zwischen den unterschiedlichen Futterkonserven, insbesondere zwischen Heu und Heulagen, nicht analysiert werden. Studien mit kolon-fistulierten Pferden belegen, dass weder unmittelbar nach der Umstellung der Fütterung von Heu auf Grassilage oder Heulage noch bei längerfristiger Fütterung dieser beiden Futtermittel nennenswerte Veränderungen in der Mikroflora 
- Tab. 3 Nährstoffgehalte und Fermentationsprofile in Heu, Heulage und Grassilage (Trockensubstanz Angabe in Prozent, weitere Angaben in g pro kg Trockensubstanz) [4].

\begin{tabular}{|l|c|c|c|}
\hline Parameter & Grassilage & Heulage & Heu \\
\hline Trockensubstanz (\%) & 34 & 55 & 86 \\
\hline Rohprotein & 176 & 151 & 165 \\
\hline wasserlösliche Kohlenhydrate & 80 & 118 & 116 \\
\hline pH & 4,4 & 5,8 & 5,9 \\
\hline kurzkettige Fettsäuren, gesamt & 51 & 2,6 & 1,6 \\
\hline Laktat & 43 & 1,3 & 0,3 \\
\hline
\end{tabular}

- Tab. 4 Fermentationsprofile im Koloninhalt von Pferden nach Fütterung von Heu, Heulage oder Grassilage (Angaben in mmol/l Kolon-Chymus) [4].

\begin{tabular}{|l|c|c|c|}
\hline Parameter Koloninhalt & Grassilage & Heulage & Heu \\
\hline pH & 6,81 & 6,74 & 6,75 \\
\hline Laktat & 0,16 & 0,08 & 0,07 \\
\hline kurzkettige Fettsäuren, gesamt & 60 & 58 & 62 \\
\hline kurzkettige Fettsäuren, Acetat & 43 & 47 & 48 \\
\hline
\end{tabular}

noch in dem Dickdarmmilieu, wie z. B. pH-Werte, zu finden sind [4] ( Tab. 4).

Merke
Die häufig postulierte Azidierung des Darmmilieus
durch die Fütterung von Grassilage oder Heulage
kann durch Untersuchungen nicht bestätigt werden.

\section{Haltbarkeit?}

Problematisch gestaltet sich teilweise die geringe Haltbarkeit der Heulagen nach der Öffnung der Folie. Durch die Zufuhr von Sauerstoff kann eine massive Vermehrung von Mikroorganismen, insbesondere Hefen, stattfinden, sodass bei warmen Außentemperaturen eine zügige Verfütterung (innerhalb von 12-24 h) zu empfehlen ist. Im Winter ist dieses Problem weniger prägnant, sodass u. U. die Fütterung eines geöffneten Heulageballens über 3-5 Tage möglich ist [5].

Das Problem der Großballen (250-500 kg) wurde aber in den letzten Jahren durch das Angebot von kleinen Heulageballen (10-20 kg) gelöst, sodass selbst bei der Fütterung von Einzeltieren der Verbrauch der Heulage nach dem Öffnen innerhalb von 1 Tag möglich ist.

\section{Botulismus-Risiko?}

Generell wird bei der Fütterung von Heulage oder Grassilage ein höheres Botulismus-Risiko postuliert. Aus den Fallbeschreibungen in der Literatur fallen aber auch Pferde, die mit Wiesen- oder Luzerneheu gefüttert wurden, ebenfalls häufig mit Botulismus auf. Vielfach werden die Futterqualitäten bei dem Auftreten von Botulismus im Pferdebestand als „verdorben“ beschrieben, sodass eine hygienisch einwandfreie Futtermittelqualität, unabhängig von der Konservierung, eine unabdingbare Grundvoraussetzung darstellt.

Beim Botulismus handelt es sich um eine i.d. R. tödlich verlaufende Intoxikation durch das von Clostridium botulinum (oder anderen Clostridien spp.) gebildete Botulinustoxin. Die Toxinbildung findet unter Luftabschluss statt, z. B. in in Folien abgepackten Silagen oder in verklumpten Nestern im Heu.

In das Futter gelangen Clostridien vor allem über Erde oder über tote Tiere während der Ernte, z.T. aber auch während einer unsachgemäßen Lagerung des Futters. Um das Botulismusrisiko deutlich eindämmen zu können, ist es wichtig, Gräser mehrere Zentimeter oberhalb der Grasnarbe zu schneiden, um einen Erdeintrag in das Futter zu vermeiden. Zudem sollte das Einbringen von Tieren vermieden und auf eine adäquate Futterlagerung geachtet werden.

\section{Freies Kotwasser?}

Weitere Nachteile bei der Fütterung von Heulagen oder Grassilagen liegen in der Problematik des freien Kotwassers. Die Ursachen für das Auftreten von freiem Kotwasser sind bislang nicht eindeutig geklärt. Aus eigenen Beobachtungen gibt es aber zahlreiche Fälle, die bei der Fütterung von Heu (hier vor allen Dingen sehr überständige bzw. schilfartige Gräser) auftreten, sodass dies nicht ausschließlich ein Phänomen der Heulagefütterung ist.

\section{Hafer und Co}

Gequetschter Hafer weist deutlich höhere Gehalte an respiratorischen Partikeln auf als ungequetschte sowie extrudierte Getreide (Wasserdampf plus Druck) oder auch pelletierte Ergänzungsfuttermittel, sodass diese bevorzugt zu füttern sind. Eine ganz erhebliche Reduktion der Staubentwicklung erreicht man auch durch die Zulage von Pflanzenöl oder Melasse zum Getreide oder Ergänzungsfutter, wobei Mengen zwischen 3-5\% einzumischen sind, um eine angemessene Staubbindung zu erreichen.

\section{Supplemente}

\section{Omega-3-Fettsäuren}

Omega-3-Fettsäuren, wie sie z. B. in Lein- oder Fischöl enthalten sind, besitzen anti-inflammatorische Eigenschaften. Die mehrwöchige Zulage von Omega-3-Fettsäuren (Dosierung Docosahexaensäure: 1,5-5,3 mg/kg KM) führte zu einer Reduktion der Leukozyten in der broncho-alveolären Flüssigkeit bei chronisch lungenkranken Pferden [6, 7], wohingegen weitere Effekte, wie z. B. 
die Verbesserung von Lungenfunktionsparametern, sehr unterschiedlich ausfielen.

Generell scheint die Supplementierung von Omega-3Fettsäuren als ergänzende Maßnahme in Kombination mit einer staubreduzierten Fütterung und Haltung Potenzial zu besitzen, allerdings liegen keine hinreichenden Informationen zur Dosierung und Behandlungsdauer vor.

\section{Antioxidantien}

Der Ergänzung von Antioxidantien, wie z.B. Vitamin E oder Vitamin C, als Begleittherapie zur staubarmen Fütterung und Haltung wird vielfach diskutiert. Durch die Zulage von Vitamin-E- und Vitamin-C-reichen Supplementen können die entsprechenden Plasmaspiegel signifikant erhöht werden, Konsequenzen für die Entzündungsbereitschaft oder für die Lungenfunktion konnten aber bei Pferden, die an Equinem Asthma litten, nicht beobachtet werden $[8,9]$.

Eine bedarfsangepasste Nährstoffempfehlung ist grundsätzlich bei Pferden mit Equinem Asthma anzuraten, über den Bedarf hinausgehende Supplementierungseffekte von Vitamin E, Vitamin C oder auch Selen sind aber nach aktuellem Kenntnisstand nicht zu erwarten.

\section{Gegen Husten ist kein Kraut gewachsen}

Unbestritten ist, dass es zahlreiche Kräuter gibt, die beispielsweise eine schleimlösende Wirkung besitzen oder das Immunsystem unterstützen können.

Die Fütterung eines kommerziellen Kräutergemischs mit einem hohen Anteil an Flavonoiden (wie z.B. Quercetin, Azaleatin, Rution und Kampfer, Konzentrationen nicht spezifiziert) führte bei konventioneller Haltung und Fütterung ohne Maßnahmen zur Staubreduktion allerdings zu keiner wesentlichen Verbesserung der klinischen Symptomatik sowie bei den Lungenfunktionsparametern bei Pferden mit Equinem Asthma [10]. Die wenigen weiteren Studien, die bei Pferden zum Einsatz von Kräutern zur Behandlung von chronischen Atemwegserkrankungen vorliegen, zeigen ähnliche Ergebnisse, sodass aktuell zum Einsatz von Kräutern keine Empfehlung auszusprechen ist.

\section{Fazit}

Im Vordergrund bei der Prävention und Therapie von chronischen Atemwegserkrankungen (syn. Equines Asthma) stehen eine möglichst staubfreie Fütterung und Haltung. Als optimal gilt in vielen Fällen die ganzjährige 24-stündige Weidehaltung, was allerdings auf Schwierigkeiten und Grenzen stoßen kann. Im Hinblick auf die Schadwirkung von Futtermitteln auf den Respirationstrakt können verschiedene Faktoren wie z.B. die Ein- atmung von schimmelhaltigem Staub bei der Fütterung oder durch die Einstreu von Bedeutung sein, aber auch erhöhte Ammoniakgehalte in der Stallluft können den Atmungstrakt schädigen.

Maßnahmen wie das Wässern oder Bedampfen des Heus führen zu einer signifikanten Reduktion der lungengängigen, inhalierbaren Staubpartikel, aber auch die Fütterung von Heulagen trägt zur deutlichen Reduzierung des inhalierbaren Staubs bei. Bei der Kraftfutterfütterung können staubreduzierende Maßnahmen, wie z. B. die Fütterung von pelletierten Ergänzungsfuttermitteln oder das Einmischen von Pflanzenölen einen sinnvollen Beitrag leisten. Die Fütterung von Supplementen, wobei vielfach Kräuter, aber auch immunstimulierende Ergänzungen wie Omega-3-Fettsäuren auf dem Markt zu finden sind, erfreuen sich darüber hinaus großer Beliebtheit, wobei hier allerdings der Nutzen zur Prävention und Therapie bei chronischen Atemwegserkrankungen kritisch zu hinterfragen ist.

\section{Korrespondenzadresse}

PD Dr. med. vet. Ingrid Vervuert

Universität Leipzig

Veterinärmedizinische Fakultät

Institut für Tierernährung, Ernährungsschäden und Diätetik

An den Tierkliniken 9

04103 Leipzig

ingrid.vervuert@vetmed.uni-leipzig.de

Literatur

[1] VDLUFA 2011. Verfahrensanweisung zur mikrobiologischen Qualitätsbeurteilung. Methodenbuch III, 8. VDLUFA Verlag

[2] Moore-Colyer MJS, Taylor JLE, James R. The effect of steaming and soaking on the respirable particle, bacteria, mould and nutrient content in hay for horses. J Equine Sci 2016; 39: 6268

[3] Moore-Colyer MJS, Payne V. Palatability and ingestion behaviour of 6 Polo ponies offered a choice of dry, soaked and steamed hay for 1 hour on three seperate occasions. Adv Animal Biosci 2012; 3: 127

[4] Julliand V, Lindberg JE, Bertilsson J, Jansson A. Effects on the equine colon ecosystem of grass silage and haylage diets after an abrupt change from hay. J Anim Sci 2009; 87(7): 22912298

[5] Vervuert I. Welche Risiken bergen Heulagen oder Grassilagen für Pferde? Prakt Tierarzt 2015; 96(2): 159-162

[6] Khol-Parisini A, van den Hoven R, Leinker S, Huland HW, Zentek J. Effects of feeding sunflower oil or seal blubber oil to horses with recurrent airway obstruction. Canadian J Vet Res 2007; 71: 59-65

[7] Nogradi N, Couetil LL, Messick J, Stochelski MA, Burgess JR. Omega-3 fatty acid supplementation provides an additional benefit to a low-dust diet in the management of horses with chronic lower airway inflammatory disease. J Vet Intern Med 2015; 29(1): 299-306

[8] Deaton CM, Marlin DJ, Smith NC, Harris PA, Schroter RC, Kelly FJ. Antioxidant supplementation in horses affected by recur- 
rent airway obstruction. Nutr 2004; 134 (8 Suppl): 2065S20675

[9] Kirschvink N, Fiévez L, Bougnet V, Art T, Degand G, Smith N, Marlin D, Roberts C, Harris P, Lekeux P. Effect of nutritional antioxidant supplementation on systemic and pulmonary antioxidant status, airway inflammation and lung function in heaves-affected horses. Equine Vet J 2002; 34(7): 705-712

[10] Pearson W, Church A, Brewer D, Clarke AF. Pilot study investigating the ability of an herbal composite to alleviate clinical signs of respiratory dysfunction in horses with recurrent airway obstruction. Canadian J Vet Res 2007; 71: 145-151
Bibliografie

DOI https://doi.org/10.1055/a-0579-8796

Pferdespiegel 2018; 21: 107-114

(c) Georg Thieme Verlag KG Stuttgart · New York ISSN 1860-3203 